\title{
Meeting report \\ MicroRNAs and cancer: what we know and what we still have to learn
}

\author{
George A Calin
}

Address: Department of Experimental Therapeutics and Cancer Genetics, University of Texas, MD Anderson Cancer Center, Houston, TX 77030, USA. Email: gcalin@mdanderson.org

\begin{abstract}
A report on the Keystone Symposia on MicroRNAs and Cancer, Keystone, Colorado, USA, 10-15 June 2009.
\end{abstract}

\section{Introduction}

The organizers of the 2009 Keystone Symposia on MicroRNAs and Cancer assembled a fine panel of speakers, and the audience of more than 300 participants consisted mainly of young scientists, including graduate students, postdoctoral fellows and early career researchers, all fully convinced that the microRNA (miRNA) revolution in the field of cancer research has already arrived! The meeting had double the number of delegates of the previous meeting two years ago, proof that the scientific community is focused on understanding the roles of these small regulatory RNAs in human tumors. The meeting was organized in plenary sessions centered on the main research avenues, such as 'Regulation of development by miRNAs', 'Gene regulation by non-coding RNAs', 'MicroRNAs in cancer', 'Roles of small RNAs in cancer', and 'MicroRNAs as diagnostics and therapeutics'. In addition, workshops were held based on the most interesting posters and focusing on new topics related to clinical translation, such as 'Bench to bedside: translating miRNA discoveries into clinical applications', 'Hot topics in miRNA research' or 'Emerging ideas and technologies in small RNAs'. As proved by the previous enumeration, two new research directions were wisely selected by the organizers to be presented and discussed extensively - firstly, the clinical applications of the already overwhelming number of mechanistic discoveries related to miRNAs and, secondly, the involvement of other short or long non-coding RNAs (ncRNAs) in human cancers and their potential use for patients. Clearly proving this research tendency was the selection, as Keynote address, of the seminal contribution of Joan Massague (Memorial Sloan-Kettering Cancer Center, New York, NY, USA) on understanding the mechanisms of metastasis and of miRNA involvement in late-stage tumorigenesis.

\section{Bench to bedside - microRNAs are already there!}

The translational implications of miRNA research for cancer patients certainly represents one of the most exciting avenues of research due to its great diagnostic, prognostic and therapeutic implications. Curtis C Harris (National Institute of Health, MD, USA) presented exciting new data, as well as previously published work, proving that miRNAs can be used as biomarkers of diagnosis, prognosis and therapeutic outcome in lung, colon and esophageal cancers all tumors with high incidence and high mortality. Frank Slack (Yale University, CN, USA) presented exciting results showing miR-34 effects linked to the response to radiation therapy in triple negative (estrogen receptor (ER)-, progesterone receptor (PR)- and human epidermal receptor 2 (HER2)-) breast cancer patients. Joshua Mendell (Johns Hopkins University, MD, USA) described the roles of Myc and p53 in the regulation of miRNA expression and the therapeutic consequences of restoring $m i R-26 a$ in hepatocellular carcinomas; data produced in his and in Jerry Mendell's laboratories were published in Cell on the same day as the presentation. Carlo Croce (Ohio State University, $\mathrm{OH}, \mathrm{USA}$ ), whose pioneer work in 2002 initiated the field of microRNAs and cancer, elaborated on the mechanisms of miRNA alterations in human cancers, including gene deletions and germline mutations, and expanded the new concept that miRNAs are useful predictors of metastatic potential and of response to therapy. Joanne Weidhaas (Yale University School of Medicine, CN, USA) presented the exciting findings that single nucleotide polymorphisms in a target site of KRAS for let-7 represent a biomarker for cancer risk, including cancers of the lung and breast. David Brown (Asuragen, TX, USA) presented the efforts of his team in their work to develop therapeutic applications for tumor suppressor miRNAs. A number of short talks during workshops specifically addressed the issue of translational research; among these, Dalia Cohen's talk (Rosetta Genomics, PA, USA) concerned the use of a miRNA signature that can identify tumor tissue origin, whereas Helen Henegen's

ER, estrogen receptor; HER2, human epidermal receptor 2; miRNAs, microRNAs; ncRNAs, non-coding RNAs; PR, progesterone receptor; RNAi, RNA interference; TRBP, TAR RNA-binding protein 2; UTR, untranslated region. 
(National University of Ireland Galway, Ireland) presentation was related to plasma miRNAs as biomarkers for breast cancer.

\section{Understanding the mechanisms of miRNA dysregulation in human cancers}

This represents an essential aspect of miRNA research, as understanding what causes aberration in miRNA expression to occur in cancer is helpful not only for oncology, but also for understanding the roles of miRNAs during normal development and differentiation. Scott Hammond (University of North Carolina, Chapel Hill, NC, USA) presented evidence of a new mechanism that could explain the downregulation of suppressor miRNAs in human cancer, represented by Lin-28 regulation of RNase III Drosha. Sonia Mello (Catalan Institute of Oncology, Barcelona, Spain) presented an additional mechanism for impairment of microRNA processing and Dicer function in sporadic and hereditary carcinomas with microsatellite instability - the truncating mutations in TAR RNA-binding protein 2 (TRBP). Bringing a completely new and highly needed expertise in the young field of miRNAs in cancer, RNA crystallography, Jennifer Doudna (Genentech, CA, USA) presented her lab's efforts to identify the architecture of human Dicer and partner proteins. Marianthi Kiriakidou (University of Pennsylvania School of Medicine, PA, USA) focused on the roles of another protein involved in miRNA processing, Argonaute 2, and uncovered expression data for Ago2 in various human cancers.

\section{Dissecting the roles of microRNAs in human cancers}

This represents the mainstream of cancer research related to miRNAs - what are the functional consequences of miRNA alterations in human cancers? Amy Pasquinelly (University of California at San Diego, CA, USA) presented the current efforts in her laboratory, based on a Caenorhabditis elegans model, to understand the regulation of gene expression by direct interaction of miRNAs. Pasquinelly found 'non-classical' miRNA-target interactions involving regions of messenger RNA outside the 3' untranslated region (UTR). On the same line of evidence, Isidore Rigoutsos (IBM) presented new data proving the importance of $5^{\prime}$-UTR interaction for biological processes with a wider significance for body homeostasis, such as iron metabolism. Judy Lieberman (Harvard Medical School, MA, USA) talked about the roles of $m i R-24$ in targeting multiple genes from signaling pathways that are important for breast cancer. Alexander Schier (Harvard University, MA, USA) linked the roles of miR-43O in development with the potential roles of this miRNA in metastases. Brian Cullen (Duke University Medical Center, NC, USA) elaborated on the roles of viral miRNAs in cancer and on the interdependent alteration of cellular miRNAs such as $m i R-155$, one of the most frequent dysregulated oncogenes in human cancers. Eric Lai (Memorial SloanKettering Institute, NY, USA) emphasized the interrelation between WNT and Hippo pathways, both significantly altered in various types of human cancers, and miRNAs, while Elaine Fuchs (Rockefeller University, NY, USA) described miRNA-dependent fine regulation of the balance between growth and differentiation in skin stem cells. Carl Novina (Harvard University, MA, USA) explored the tumor suppressive roles of $m i R$-211 in melanoma, while Eric Miska (University of Cambridge, UK) detailed a new quantitative and high-throughput in vivo assay for miRNA function in C. elegans.

\section{Other non-coding RNAs and cancer}

While the main focus at present is to understand the roles of miRNAs in cancer, there is still a lot of effort aimed at deciphering the complex ncRNA network, including short and long ncRNAs, which is, overall, altered in cancers. Following this line of research, John Mattick (University of Queensland, Australia) elaborated on the roles of ncRNAs in evolution and development, and revealed new data related to the existence of $3^{\prime}$-UTR non-coding transcripts independent of host protein-coding gene transcripts. I included in my presentation data regarding the roles of long ultraconserved ncRNAs in human cancers and their potential use as diagnostic and prognostic markers. Mark Kay (Stanford, CA, USA) presented a fine dissection on the roles of small RNAs in gene regulation, while Yong Sun Lee (University of Texas, Medical Branch, TX, USA) emphasized the roles of a new class of small RNAs, the tRNAderived RNA fragments in cell proliferation. Using C. elegans as a model, Alla Grishok (Columbia University Medical Center, NY, USA) talked about gene expression regulation by endogenous RNA interference (RNAi) and retinoblastoma tumor suppressor.

As happens in every 'MicroRNAs and Cancer' Keystone Symposium, the interconnections between the various talks and the ideas presented in both posters and during free discussions were very high; the audience certainly left not only with answers to some questions, but also with new questions to be answered that will undoubtedly lead to the development of alternative lines of research. This is indeed an exciting time, as the ncRNA revolution in cancer research is shifting the classic paradigms.

\section{Competing interests}

The author declares that he has no competing interests.

Published: 17 August 2009

doi:10.1186/gm78

(C) 2009 BioMed Central Ltd 